\title{
Distúrbios Cardiovasculares na Acromegalia
}

revisão

\section{DANIELA FEDRIZZI \\ mauro antonio Czepielewski}

Unidade de

Neuroendocrinologia do Serviço

de Endocrinologia do Hospital de Clínicas de Porto Alegre;

Programa de Pós-Graduação em Ciências Médicas:

Endocrinologia, Metabolismo e Nutrição da Faculdade de Medicina da Universidade Federal do Rio Grande do Sul (UFRGS), Porto Alegre, RS, Brasil.

A acromegalia acarreta uma série de distúrbios ao sistema cardiovascular, decorrentes da exposição crônica a níveis elevados de GH e IGF-1. Estes distúrbios são os principais responsáveis pelo aumento da mortalidade de acromegálicos. Entre as várias formas de acometimento cardiovascular, destaca-se a miocardiopatia acromegálica, entidade caracterizada, inicialmente, pelo estado hiperdinâmico, seguido de hipertrofia ventricular esquerda concêntrica e disfunção diastólica por déficit de relaxamento, culminando com disfunção sistólica e, por vezes, insuficiência cardíaca franca. Além disso, são também relevantes as arritmias, as valvulopatias, sobretudo mitral e aórtica, a cardiopatia isquêmica, a hipertensão e os distúrbios dos metabolismos glicêmico e lipídico. Nesta revisão são abordados os principais aspectos clínicos e prognósticos destas entidades, os efeitos do tratamento da acromegalia sobre elas e as repercussões correspondentes sobre a sobrevida dos pacientes. (Arq Bras Endocrinol Metab 2008; 52/9:1416-1429)

Descritores: Acromegalia; Miocardiopatia acromegálica; Hipertensão secundária; Risco cardiovascular

\section{ABSTRACT}

\section{Cardiovascular Disturbances in Acromegaly.}

Acromegaly causes a number of disorders in the cardiovascular system, resulting from chronic exposure to high levels of GH and IGF-1. Such disorders are the main responsible for increased mortality rates among acromegalic patients. Among several forms of cardiovascular impairment is acromegalic cardiomyopathy, an entity that is initially characterized by a hyperdynamic state, followed by concentric left ventricular hypertrophy and diastolic dysfunction due to relaxation deficit, culminating in systolic dysfunction and sometimes heart failure. In addition, arrhythmias and heart valve diseases are also relevant, especially mitral and aortic, ischemic heart disease, hypertension, and glucose and lipid metabolism disorders. This review approaches the main clinical and prognostic aspects of these entities, the effects of acromegaly treatment on them, and the respective consequences on patient survival. (Arq Bras Endocrinol Metab 2008; 52/9:1416-1429)

Keywords: Acromegaly; Acromegalic cardiomyopathy; Secondary hypertension; Cardiovascular risk

\section{INTRODUÇÃO}

cromegalia é a doença endócrina resultante do excesso crônico de hormônio do crescimento $(\mathrm{GH})$, em virtude, na maioria dos casos, de ademilhão e incidência estimada em três a quatro casos por milhão de pessoas 
por ano (1). Homens e mulheres parecem ser igualmente afetados e a idade média de apresentação da doença situa-se entre 40 e 50 anos (1).

A acromegalia pode ser causada por tumores hipofisários ou por doenças extra-hipofisárias (2). Independentemente da etiologia específica, a doença caracteriza-se por níveis elevados de GH e IGF-1, com conseqüentes sinais e sintomas decorrentes do hipersomatotropismo (2). Os efeitos do excesso de GH e IGF-1 sobre o crescimento acral e visceral e sobre o metabolismo ocorrem insidiosamente. Os efeitos somáticos mais conhecidos são: aumento de extremidades e de partes moles, desfiguração facial, hiper-hidrose e artropatias. Além disso, há uma série de efeitos sistêmicos já bem caracterizados, entre os quais se destacam as alterações do metabolismo da glicose, dos sistemas cardiovascular e respiratório e o aumento da incidência de neoplasias de cólon. Por causa de seu curso indolente, tanto a acromegalia em si quanto suas repercussões clínicas podem sofrer considerável atraso no seu diagnóstico e tratamento (3).

O hipersomatotropismo crônico acarreta importantes alterações estruturais e funcionais sobre o sistema cardiovascular. De fato, as mortes cardiovasculares são as principais contribuintes para o aumento de mortalidade na acromegalia (3-10). No recente estudo do Registro Espanhol de Acromegalia ocorreram 56 mortes em 1.219 pacientes, sendo a maioria delas de etiologia cardiovascular (9). $\mathrm{Na}$ época do diagnóstico, arritmias, hipertensão e doenças valvulares estavam presentes em até $60 \%$ dos pacientes (4). Com a progressão da doença, pode ocorrer a evolução para hipertrofia miocárdica concêntrica e insuficiência cardíaca diastólica (4). Cerca de metade dos pacientes com acromegalia ativa é hipertensa, e metade destes tem evidências de disfunção ventricular esquerda (2). Curiosamente, a hipertrofia ventricular esquerda também é descrita em cerca de metade dos pacientes acromegálicos normotensos (2). A coexistência de hipertensão e doença arterial coronariana responde pela maior parte do acometimento cardiovascular encontrado nos pacientes com acromegalia. Também costuma estar presente miocardiopatia específica da doença, mediada pelo excesso de GH e IGF-1 (2). O controle efetivo dos níveis destes hormônios resulta melhora global da função cardiovascular.

Não obstante o muito que já se sabe sobre as repercussões da acromegalia no sistema cardiovascular, diversos aspectos continuam suscitando controvérsia.
O progresso das modalidades de tratamento da acromegalia se fez acompanhar de redução de sua mortalidade, embora ainda seja elevado o percentual de óbitos e complicações por problemas cardíacos decorrentes da acromegalia. Aparentemente, a despeito da melhora dos índices de cura e da busca de parâmetros mais rigorosos de controle bioquímico, não parece estar ocorrendo diminuição proporcional dos distúrbios cardiovasculares. Isso sugere que há outros fatores implicados na patogênese destes distúrbios, além do aumento do GH e do IGF-1, talvez com igual ou até maior impacto do que os níveis hormonais propriamente ditos. Recente e extensa metanálise demonstra que o aumento da mortalidade por todas as causas em pacientes acromegálicos é achado consistente de mais de 16 estudos selecionados na literatura (11). Mesmo entre pacientes tratados com cirurgia transesfenoidal, a mortalidade persiste elevada (11). Há de se considerar, ainda, a imensa heterogeneidade que certamente existe entre os pacientes que foram considerados "curados", pois os diversos métodos para dosagem de $\mathrm{GH}$ atualmente disponíveis podem levar a interpretações equivocadas, uma vez que a especificidade dos ensaios é muito diferente (12).

Diante do exposto, o objetivo desta revisão é examinar os aspectos mais importantes das complicações cardiovasculares dos pacientes acromegálicos, bem como suas relações com a progressão e o tratamento da doença. São abordados a patogênese, as manifestações clínicas e os efeitos do tratamento da acromegalia sobre a miocardiopatia acromegálica, as valvulopatias, as arritmias, a cardiopatia isquêmica, a hipertensão e as alterações dos metabolismos glicêmico e lipídico. Assim, esta revisão pretende chamar a atenção para o fato de que o manejo do paciente acromegálico deve incluir o controle de suas diversas alterações clínicas e os distúrbios associados, além dos clássicos parâmetros hormonais (GH e IGF-1).

\section{MIOCARDIOPATIA ACROMEGÁLICA}

Atualmente, é consenso entre os autores a existência de miocardiopatia específica da acromegalia, que é parcialmente reversível com a diminuição dos níveis de $\mathrm{GH}$ e IGF-1 (6). A fibrose intersticial é o principal achado, similar à miocardite multifocal, porém sem necrose $\mathrm{e}$ com aumento da apoptose dos miócitos cardíacos (6). Cerca de $20 \%$ dos acromegálicos jovens (abaixo dos 30 anos) e normotensos têm hipertrofia cardíaca (13). Em pacientes com menos de 40 anos de idade e com acro- 
megalia ativa por 3 a 7 anos, 54\% tinham evidências ecocardiográficas de hipertrofia ventricular esquerda, aumentando para $72 \%$ em pacientes entre 41 e 60 anos e com maior duração de doença (14). Ou seja, a hipertrofia cardíaca sem dilatação ventricular é achado comum e precoce na acromegalia.

A primeira descrição da miocardiopatia acromegálica como entidade clínica distinta foi de Sacca e cols., que descreveram sua evolução em três estágios: precoce, típica de pacientes jovens e com curta duração, caracterizada por freqüência cardíaca elevada e aumento do débito sistólico; intermediária, caracterizada por hipertrofia cardíaca e disfunção diastólica; e tardia, com diminuição da função sistólica, culminando em insuficiência cardíaca, se o excesso hormonal não for tratado adequadamente $(14,15)$.

A fibrose intersticial constitui a principal anormalidade histológica encontrada na miocardiopatia acromegálica (3). Subseqüentemente, ocorre desarranjo gradual da arquitetura cardíaca por meio da deposição de colágeno extracelular, desorganização miofibrilar e áreas de infiltrado linfomononuclear, caracterizando padrão de miocardite (3). Em estudo de biópsias de tecido cardíaco, obtidas durante cateterismos feitos em pacientes acromegálicos, detectou-se aumento da apoptose dos miócitos cardíacos e fibroblastos intersticiais, inversamente correlacionado ao débito cardíaco $(3,14)$.

A característica mais importante da chamada miocardiopatia acromegálica é a hipertrofia cardíaca. Embora a hipertrofia de ventrículo esquerdo seja o achado clássico, o ventrículo direito pode estar igualmente envolvido (14). Mesmo utilizando-se métodos de correção da massa ventricular (altura, superfície corporal), a prevalência de hipertrofia ventricular esquerda em acromegálicos persiste elevada (16). A idade e o tempo de duração da acromegalia são os principais determinantes do acometimento cardíaco. Dados de autópsias e in vivo dão conta de freqüência de hipertrofia cardíaca de mais de $90 \%$ em pacientes com doença de longa duração (6). Em pacientes mais jovens e com menor duração da acromegalia, pode igualmente ocorrer hipertrofia cardíaca, como já foi mencionado anteriormente. Em pacientes com duração estimada da acromegalia de menos de 5 anos, a massa do ventrículo esquerdo era 36\% maior do que a dos controles (17). Outro grupo, estudando pacientes acromegálicos cujo critério de seleção era a idade cronológica (menos de 30 anos), encontrou maiores índices de massa ventricular esquerda e maior incidência de déficit de relaxamento nos pacientes do que em controles pareados pela idade (13). Tais achados sugerem que a hipertrofia cardíaca seja evento precoce na acromegalia, e que piore proporcionalmente com a duração da atividade da doença.

Casini e cols. (18) analisaram 40 pacientes acromegálicos, com o objetivo de avaliar os fatores determinantes da hipertrofia ventricular esquerda, concluindo que a presença de hipertensão arterial e os níveis de IGF-1 foram os principais determinantes da hipertrofia. Lopez-Velasco e cols. (19), estudando alterações cardíacas em acromegálicos por meio de ecodopplercardiografia, encontraram aumento de massa ventricular esquerda, débito cardíaco e tempo de relaxamento isovolumétrico, independentemente da presença ou da ausência de hipertensão arterial. Estes achados corroboram o papel da miocardiopatia acromegálica na patogênese das alterações morfofuncionais cardíacas destes pacientes.

A hipertrofia cardíaca está associada a alterações funcionais, entre as quais se destaca a diminuição da capacidade de enchimento ventricular (3). Estudos com ecodopplercardiografia documentaram diminuição da onda de enchimento diastólico e aumento do tempo de relaxamento isovolumétrico $(6,14)$. Esses distúrbios podem permanecer assintomáticos por vários anos. Além disso, na presença de disfunção diastólica, a recuperação incompleta de pré-carga adequada pode afetar os parâmetros sistólicos durante esforço físico $(6,14)$. Colao e cols. (3) ao estudarem coorte de acromegálicos normotensos e sem diabetes encontraram associação inversa entre função diastólica e tempo estimado de duração da doença. Isso confirma que a exposição prolongada ao excesso de GH/IGF-1 deteriora o desempenho cardíaco, mesmo na ausência de hipertensão e diabetes (3).

Considerando a miocardiopatia acromegálica como complicação da acromegalia, pode-se inferir que o tratamento da doença de base deve reverter ou ao menos minorar as manifestações sobre o miocárdio. Diversos estudos apontam esta direção. Dados epidemiológicos mostram que o sucesso do tratamento da acromegalia está associado à redução consistente da morbimortalidade cardiovascular, seja este tratamento feito por cirurgia, radioterapia, análogos de somatostatina ou associações entre estes métodos. Observam-se mudanças estruturais no coração com a adequada redução dos níveis de GH e a normalização do IGF-1 (14). A maior parte dos estudos que chegaram a esses resultados eram de curta duração (de 2 a 12 meses) e usaram análogos da somatostatina. A adenomectomia melhorou a taxa 
de mortalidade entre pacientes, nos quais se alcançou a remoção completa do tumor ou o controle bioquímico com o tratamento adjuvante pós-cirúrgico (20). Por outro lado, um grupo de pacientes submetidos à radioterapia mostrou piora das alterações ecográficas e eletrocardiográficas durante o seguimento a longo prazo (21). Aqui cabe a ressalva de que este grupo foi avaliado na década de 1980, quando os critérios de cura da acromegalia eram diferentes, portanto alguns deles poderiam estar ainda com níveis hormonais que hoje seriam considerados doença em atividade. Assim, vários achados sugerem que a melhora dos parâmetros cardiovasculares depende do sucesso do tratamento empregado.

O remodelamento cardíaco nos pacientes tratados parece ocorrer rápida e significativamente. Colao e cols. (22) descrevem a redução de massa ventricular esquerda em pacientes de todas as idades, bem como o retorno a índices de massa ventricular normais em metade dos pacientes estudados. A remoção do adenoma somatotrófico resultou em melhora da massa ventricular e do desempenho diastólico em um grupo de 30 pacientes acromegálicos virgens de tratamento (23). Em uma coorte de 18 pacientes curados acompanhados por cinco anos, houve significativa melhora da fração de ejeção durante o esforço e da freqüência cardíaca em repouso, independentemente do método de tratamento empregado (cirurgia versus cirurgia mais análogo da somatostatina) (22). A análise cardíaca por ecorrefringência, que é medida indireta da fibrose miocárdica, apresentou reversão dos índices a parâmetros normais após a cura da acromegalia (24).

Entretanto, a recuperação da função cardíaca não depende apenas do controle bioquímico estrito da acromegalia, mas também da idade dos pacientes e do tempo de duração do hipersomatotropismo anterior à intervenção. Em um grupo de 22 acromegálicos adequadamente controlados, houve reversão da hipertrofia ventricular esquerda em todos os pacientes do grupo mais jovem e em apenas metade dos pacientes com mais de 40 anos de idade (25). É importante ressaltar que neste estudo não foram incluídos pacientes hipertensos.

Uma metanálise de estudos com análogos da somatostatina avaliou o impacto desse tratamento sobre uma série de parâmetros cardíacos (26), relacionados direta ou indiretamente à miocardiopatia acromegálica. Encontrou-se redução da freqüência cardíaca, do índice de massa ventricular esquerda, da espessura do septo interventricular e da parede posterior do ventrículo esquerdo, além de aumento da tolerância ao exercício, nos pacientes tratados.

Mais recentemente, o pegvisomanto, um antagonista do receptor de $\mathrm{GH}$, também demonstrou exercer efeitos benéficos sobre a estrutura cardíaca. Em um estudo com 17 pacientes submetidos a tratamento com pegvisomanto, o uso da droga pelo período de 18 meses mostrou diminuição do tempo de relaxamento isovolumétrico e do índice de massa ventricular esquerda, bem como aumento da fração de ejeção, refletindo melhora tanto da função diastólica quanto da função sistólica (27).

Em resumo, a miocardiopatia acromegálica é complicação freqüente da acromegalia não controlada, tem espectro variado de manifestações clínicas e pode evoluir para disfunções diastólica e sistólica. O controle agressivo da hipersecreção de GH/IGF-1 - ressecção do adenoma hipofisário, tratamento medicamentoso ou radioterapia, isolados ou associados - pode minimizar ou até mesmo reverter a disfunção cardíaca decorrente desta miocardiopatia.

\section{ARRITMIAS}

Comparadas às alterações estruturais e funcionais, as alterações do ritmo cardíaco são menos documentadas (14). Sabe-se que a freqüência e a gravidade das arritmias ventriculares são maiores em acromegálicos quando comparados aos controles; especificamente, $48 \%$ dos acromegálicos tinham arritmias ventriculares complexas, em um estudo de Kahaly e cols. (28), comparados com somente $12 \%$ dos controles. As extra-sístoles supraventriculares não parecem ocorrer mais freqüentemente em acromegálicos; ao contrário, extra-sístoles ventriculares, fibrilação atrial paroxística, doença do nó sinusal, taquicardia ventricular e bloqueios de ramo são arritmias comuns em acromegálicos, especialmente em picos de esforço físico.

Os pacientes acromegálicos podem apresentar aumento da duração do intervalo QT, o que é considerado fator de risco estabelecido para arritmias cardíacas potencialmente fatais. Fatti e cols. (29) estudaram o impacto do tratamento com análogos da somatostatina sobre a duração do QTc, encontrando redução desse intervalo a níveis comparáveis com os dos controles.

Hermann e cols. (30) avaliaram a ocorrência de potenciais ventriculares tardios na acromegalia ativa. Os potenciais tardios são ondas de baixa amplitude e alta 
freqüência na porção terminal do complexo QRS no eletrocardiograma (ECG), e são considerados preditores de eventos arrítmicos em pacientes com infarto do miocárdio prévio. Os pesquisadores encontraram potenciais tardios no ECG em 56\% dos pacientes com acromegalia ativa, em $6 \%$ dos pacientes com acromegalia controlada e em nenhum dos controles (30).

O uso do holter pode ser de importância especial na avaliação pré-operatória dos pacientes acromegálicos, já que arritmias graves podem ser causa de morte súbita na acromegalia. Um estudo multicêntrico realizado na Itália relatou a ocorrência de extra-sístoles ventriculares em 33\% dos pacientes acromegálicos avaliados por holter de 24 horas (31).

\section{VALVULOPATIAS}

A doença valvular cardíaca é importante componente da disfunção ventricular, mas as alterações das válvulas mitral ou aórtica têm sido relativamente pouco abordadas na acromegalia (32). Lie e Grossman (33) encontraram alterações mitrais e aórticas em 19\% dos casos em sua série de autópsias. Ohtsuka e cols. (34) descreveram fragilidade do anel valvar e desarranjo dos folhetos, acompanhados por regurgitação e estenose valvar. Colao e cols. (32), ao avaliarem as válvulas mitral e aórtica em 64 pacientes acromegálicos e 64 controles, encontraram prevalência mais alta de doença valvular nos pacientes (tanto curados quanto com doença ativa) do que nos controles. Entre os sujeitos que não tinham hipertrofia ventricular esquerda, no mesmo trabalho, apenas os portadores de acromegalia tinham alterações valvares (32).

Pereira e cols. (35) investigaram a prevalência de doença valvular em 40 acromegálicos $(22$ pacientes em remissão e 18 com doença ativa) e 120 controles. Por meio de ecocardiograma com Doppler, foi encontrada regurgitação aórtica em 30\% dos pacientes, contra $7 \%$ dos controles, e regurgitação mitral em $35 \%$ dos pacientes, contra $32 \%$ dos controles (35). Além disso, foi avaliada a contribuição de diversos fatores para a ocorrência de valvulopatia, sendo encontrada significância com a duração da acromegalia, mas não com os outros parâmetros avaliados, a saber: níveis de GH e IGF-1 ao diagnóstico, idade, atividade da doença, uso de octreotide e presença de hipertensão arterial (35). É interessante observar que nesse trabalho todos os pacientes com alterações valvulares tinham função ventricular esquerda normal e $85 \%$ deles tinham dimensões cardíacas também normais.
Os autores teorizaram que estes achados poderiam ter ocorrido em razão da menor duração da doença valvular nestes pacientes. Posteriormente, o mesmo grupo reavaliou os pacientes utilizando ecocardiograma com Doppler após o período de acompanhamento de um e meio até três anos, encontrando aumento significativo da ocorrência de doença valvular com o tempo (presente em $46 \%$ dos pacientes no início e em $67 \%$ no final do estudo) (36). Este aumento foi significativo apenas para a doença valvular mitral, e somente o grupo com acromegalia não-controlada apresentou aumento significativo da prevalência de valvulopatia. $\mathrm{O}$ controle adequado da secreção de GH foi associado com função valvular estável durante o tempo de seguimento do estudo (36). Entretanto, ainda não se conhece o tempo necessário de controle adequado de doença para prevenir deteriorações posteriores da doença valvular.

As valvulopatias em pacientes acromegálicos podem, em tese, decorrer do tratamento com agonistas dopaminérgicos. Nos últimos anos, surgiram diversos estudos relacionando o uso desses fármacos ao desenvolvimento de fibrose valvular. Histologicamente, as alterações descritas assemelham-se àquelas encontradas na síndrome carcinóide e no uso de derivados do ergot ou de fenfluramina. A maioria dos casos relatados ocorreu em pacientes que usavam pergolida para o tratamento de doença de Parkinson, mas já há descrições de valvulopatia com cabergolina e também com bromocriptina $(37,38)$. Até o momento, não há relatos sobre o desenvolvimento de doença valvular em pacientes usando agonistas dopaminérgicos, especificamente para o tratamento da hipersecreção de $\mathrm{GH}$, mas não há razão teórica para que isso não ocorra também neste contexto.

Em suma, a doença valvular na acromegalia é componente significativo da morbidade cardiovascular ocasionada pela doença. Parece estar relacionada predominantemente ao tempo de duração da acromegalia e acomete, em especial, a válvula mitral. Os pacientes acromegálicos necessitam avaliação cardíaca adequada, incluindo a realização de exame ecocardiográfico, para estabelecer a presença e a extensão do comprometimento valvular e instituir as medidas terapêuticas apropriadas.

\section{CARDIOPATIA ISQUÊMICA}

A doença coronariana na acromegalia ainda não está totalmente caracterizada, não obstante as evidências 
epidemiológicas apontando a cardiopatia isquêmica como um dos principais fatores de morbimortalidade da doença. Entretanto, os dados mais específicos sobre a doença coronariana são oriundos, sobretudo, de séries de autópsia. O estudo de Lie e Grossman (33), por exemplo, mostrou que, de 27 pacientes com acromegalia submetidos à autópsia, 11\% apresentavam doença arterial coronariana significativa, $15 \%$ tinham evidência de infarto do miocárdio antigo e em $24 \%$ se observou aterosclerose significativa em aorta abdominal.

O endotélio é metabolicamente ativo e tem papel fundamental na homeostase vascular por meio da liberação parácrina ou autócrina de diversas substâncias (6). O endotélio saudável modula o tônus da musculatura lisa vascular e regula vários processos aterogênicos, incluindo a adesão e a agregação plaquetária, a oxidação das LDL, a síntese de citoquinas pró-inflamatórias e a proliferação de músculo liso $(6,39)$. A disfunção da célula endotelial é hoje reconhecida como o evento desencadeante do desenvolvimento do processo aterosclerótico (39). A obesidade e a resistência insulínica estão associadas com alterações na vasodilatação mediada pelo endotélio, indicando que a obesidade está associada à disfunção endotelial. Esta última poderia, portanto, contribuir para o aumento do risco de aterosclerose em indivíduos obesos e insulino-resistentes, como aqueles portadores de acromegalia (6).

Existem alguns estudos da função endotelial na acromegalia que relatam alterações hemodinâmicas e anormalidades na função cardiovascular. Chanson e cols. (40) demonstraram a redução do fluxo na artéria braquial e o aumento da resistência vascular no antebraço em pacientes acromegálicos. Segundo os autores, a dilatação inefetiva da artéria braquial em resposta ao aumento do fluxo sanguíneo poderia ser responsável pelo aumento da resistência vascular do antebraço e poderia representar disfunção endotelial subjacente. Posteriormente, o mesmo grupo descreveu diminuição da vasodilatação dependente do endotélio, associada à resposta vasoconstritora exagerada, mediada pelo sistema nervoso simpático, em pacientes acromegálicos (41). Já o grupo de Colao, ao avaliar o fluxo sanguíneo braquial por ecografia, encontrou diminuição da dilatação mediada por fluxo na artéria braquial de pacientes acromegálicos quando comparada aos controles (42). Também está descrito menor número, redução de calibre de capilares e maior número de alças tortuosas na microcirculação capilar periférica em acromegálicos (43).
Admitindo-se as alterações endoteliais e vasculares anteriormente descritas, é de se esperar repercussões destas alterações na macrocirculação, com consequente surgimento e/ou agravamento da aterosclerose. A espessura da íntima-média da carótida foi avaliada em 45 acromegálicos, curados ou não, e em 30 controles pareados por idade e sexo, encontrando-se aumento da espessura daquela camada vascular nos pacientes, independentemente do estado bioquímico da doença (44). Entretanto, a prevalência de placas ateroscleróticas bem definidas não foi diferente entre os dois grupos (44). Estudo semelhante realizado no Japão, com 21 acromegálicos e 42 controles pareados, obteve resultado bem diferente: a média da espessura da íntima-média nos pacientes foi significativamente menor do que nos controles, enquanto 11 dos 21 pacientes acromegálicos não tinham nenhum sinal de aterosclerose (aumento da espessura da íntima-média e/ou placas ateroscleróticas visíveis) (7). Nesse estudo, os níveis de IGF-1 eram maiores e a prevalência de hipertensão arterial era menor nos pacientes sem alterações ateroscleróticas, sugerindo, segundo os autores, possível papel protetor do IGF-1 nesses pacientes.

Dados mais recentes continuam apontando a importância do acometimento coronariano e cerebrovascular na acromegalia. Uma pesquisa finlandesa publicada em 2005, por exemplo, refere como causa mortis a doença arterial coronariana em 13\% dos casos e a doença cerebrovascular em $8 \%$ dos casos, acima de outras doenças cardíacas, neoplasias e acidentes (45). Ao mesmo tempo, tem-se procurado avaliar mais diretamente a ocorrência da cardiopatia isquêmica nos acromegálicos. Dois estudos recentes avaliaram a aterosclerose em acromegálicos por meio da presença de calcificações coronarianas à tomografia computadorizada. $\mathrm{O}$ primeiro estudo, de 2006, avaliou transversalmente um grupo de 39 pacientes acromegálicos estratificados, conforme o escore de Framingham, em risco cardíaco baixo, intermediário ou alto, comparando esta classificação com o escore de cálcio coronariano (escore de Agatston) por tomografia (46). A avaliação conjunta de ambos os escores indicou que $41 \%$ dos acromegálicos estavam em risco para eventos coronarianos, e que havia calcificações coronárias evidentes em metade deles. O segundo estudo, de 2007, foi a avaliação prospectiva de desfechos semelhantes - comparação entre o escore de Framingham e o escore de Agatston e registro da ocorrência de eventos coronarianos nos 52 pacientes avaliados durante cinco anos subsequentes (47). Nesse 
estudo, $71 \%$ dos pacientes tinham risco cardíaco baixo, $27 \%$ tinham risco intermediário e $2 \%$ (um paciente) tinham risco cardíaco alto pelo escore de Framingham. Esse risco não se associou à atividade da acromegalia ou à duração da doença. O escore de Agatston também não foi associado a nenhum desses dois parâmetros. Além disso, o escore de cálcio teve papel importante apenas nos pacientes com risco intermediário, como ocorre na população geral. Os autores concluem que os acromegálicos provavelmente estão sujeitos aos mesmos fatores de risco coronarianos que os indivíduos sem acromegalia, seguindo a classificação de Framingham (47).

\section{HIPERTENSÃO ARTERIAL}

A hipertensão arterial é considerada um dos mais importantes fatores prognósticos para mortalidade na acromegalia, o que ficou definitivamente estabelecido após o trabalho de Holdaway, Rajasoorya e Gamble (10). Apesar de não ser a única responsável pelo aparecimento da disfunção ventricular e da insuficiência cardíaca na acromegalia, já que, como foi visto, existe miocardiopatia própria da doença, a coexistência de hipertensão arterial agrava os efeitos da miocardiopatia acromegálica (19). A prevalência de hipertensão nos pacientes com acromegalia varia entre $18 \%$ e $60 \%$ em diferentes séries, e a sua incidência é mais alta do que a da população geral. Em estudo com 200 acromegálicos, a prevalência de hipertensão arterial atingiu $46 \%$ dos pacientes (48). Entretanto, a despeito da sua importância, a hipertensão arterial no contexto da acromegalia ainda não tem bem esclarecidos os seus mecanismos fisiopatológicos. Verifica-se queda dos níveis pressóricos subseqüente à diminuição dos níveis de GH e IGF-1 $(3,6,49)$, o que suporta a idéia de que o hipersomatotropismo está implicado no aumento dos níveis tensionais. São diversas as possíveis explicações, incluindo alterações na regulação de sódio e água, a coexistência de alterações da homeostase glicêmica e resistência insulínica, alterações no sistema renina-angiotensina-aldosterona, distúrbios na resistência vascular periférica e alterações na ação do peptídeo natriurético atrial.

Um dos mecanismos que provavelmente contribui decisivamente para induzir hipertensão arterial na acromegalia é o aumento do volume plasmático (3). A evidência de um aumento no pool total de trocas de sódio, tanto em acromegálicos normotensos quanto hipertensos, suporta essa idéia, uma vez que existe relação dire- ta entre o pool de trocas de sódio e os níveis pressóricos (3). Supôs-se, inicialmente, que a volemia e o sódio estivessem submetidos a variações da secreção de aldosterona, mas isso não ficou claramente demonstrado na literatura, tendo sido encontrados níveis normais de aldosterona, tanto em acromegálicos normotensos quanto hipertensos (3). A atividade plasmática da renina também foi normal na maioria dos estudos realizados e não se pôde encontrar relação entre os níveis de renina e atividade da doença ou níveis de pressão arterial (3). Entretanto, mais recentemente foi descrita associação entre hipertensão arterial e polimorfismos do gene CYP11B2 em pacientes acromegálicos (49). O CYPI1B2 é um dos genes responsáveis pela transcrição da enzima aldosterona-sintetase. O polimorfismo C-344T deste gene tem sido associado com a presença de hipertensão e alterações na secreção de aldosterona em diferentes populações. Os pacientes acromegálicos com esta mutação mostraram aumento significativo da pressão sistólica (49), o que traz novamente à pauta provável papel do sistema renina-angiotensina-aldosterona na patogênese da hipertensão na acromegalia. O mecanismo proposto seria que o citado polimorfismo alteraria a taxa de transcrição da aldosterona e sensibilizaria o sistema à ação estimulatória do GH sobre a adrenal (49).

O peptídeo natriurético atrial também foi investigado como potencial causa de hipertensão arterial na acromegalia. Os níveis de peptídeo natriurético atrial foram inicialmente descritos como normais e não-correlacionados com a atividade da doença (3). Mais recentemente, as atenções se voltaram aos peptídeos natriuréticos tipo $\mathrm{B}$, secretados pelos ventrículos cardíacos em resposta a tensões da parede ventricular. Estes peptídeos vêm sendo considerados como hormônios cardioprotetores que compensam a disfunção cardíaca por meio da indução da natriurese e da modulação da hipertrofia cardíaca em resposta à expansão de volume e à distensão das paredes ventriculares. Estudo recente encontrou níveis mais baixos de peptídeo natriurético tipo B em acromegálicos, e aumento destes níveis após o controle da doença (50).

Também foi estudado o efeito da rigidez de grandes artérias sobre a hemodinâmica da circulação do paciente acromegálico. As artérias normais são estruturas complacentes, capazes de absorver as mudanças de pressão que ocorrem naturalmente durante o ciclo cardíaco. A energia é absorvida durante a sístole e liberada durante a diástole, resultando fluxo sanguíneo periférico mais constante e em manutenção da perfusão coro- 
nariana diastólica. No entanto, à medida que as artérias se enrijecem, ocorre aumento da pressão sistólica central como reflexo da maior carga imposta à periferia. A elevação da pressão central e a redução da complacência arterial levam ao desenvolvimento subseqüente de hipertrofia ventricular esquerda. A partir destes conceitos, foi avaliada a rigidez arterial em acromegálicos, tendo sido encontrado aumento da pressão aórtica central nos pacientes com doença em atividade (51).

A hipertensão arterial depende também do estado do leito vascular periférico, incluindo as arteríolas. Inicialmente, pensou-se que na acromegalia a resistência vascular sistêmica estaria diminuída, o que, associado ao aumento do índice e do débito cardíaco, poderia explicar o aumento da pressão arterial. Posteriormente, verificou-se que a resistência periférica poderia estar de fato aumentada, em decorrência de alterações morfofuncionais específicas em alguns leitos vasculares. Chanson e cols. (40) apresentaram diminuição do fluxo e aumento da resistência vascular na artéria braquial de pacientes acromegálicos, sugerindo distribuição assimétrica do débito cardíaco. Estudo com biópsias de gordura subcutânea mostrou aumento da proporção média/lúmen e aumento da espessura das paredes arteriais em nove pacientes acromegálicos, sugerindo espécie de remodelamento com hipertrofia; não houve correlação deste achado com níveis de GH (52).

Potenciais alterações do sistema adrenérgico e suas implicações na patogênese da hipertensão acromegálica também foram investigadas, com resultados conflitantes. A adrenalina e a noradrenalina plasmáticas foram descritas como normais ou aumentadas, assim como as catecolaminas urinárias. Del Rio e cols. (53) encontraram níveis de noradrenalina mais elevados em pacientes acromegálicos do que em controles, níveis esses que diminuíram durante infusão endovenosa de octreotide. Todos os pacientes desse estudo eram normotensos. Bondanelli e cols. (54) encontraram ausência do ritmo circadiano fisiológico das catecolaminas em acromegálicos, e recuperação desse ritmo após cirurgia transesfenoidal curativa.

É fundamental considerar o papel das alterações da glicemia e da sensibilidade à insulina no surgimento da hipertensão arterial na acromegalia. A capacidade do GH em antagonizar as ações da insulina é conhecida há bastante tempo, e explica porque a acromegalia é muitas vezes complicada por resistência insulínica e diabetes melito franco. O excesso de GH induz estado de resistência insulínica, manifesto inicialmente como aumento da concentração de insulina no jejum e resposta insulinêmica exagerada à sobrecarga de glicose. Permanecendo não-tratado, o excesso de $\mathrm{GH}$ provoca hiperglicemia, perda da resposta insulinêmica à sobrecarga de glicose e diminuição da insulinemia em jejum (3).

Além de seus efeitos deletérios sobre os metabolismos glicídico e lipídico, a hiperinsulinemia pode influenciar diretamente a pressão arterial por meio da retenção renal de sódio; de fato, estudos com clampes euglicêmicos associam a hiperinsulinemia à hipertensão essencial (55). Diabéticos tipo 2 com resistência insulínica grave são particularmente sensíveis aos efeitos do IGF-1 no que se refere à formação de edema, o que foi demonstrado em estudos com administração de IGF-1 recombinante (56). Há também indícios de que, em indivíduos normais, o IGF-1 pode reduzir a capacidade de excreção renal de sódio, podendo induzir edema franco em pessoas com elevada ingestão de sódio ou fluidos (56). Os pacientes acromegálicos com diabetes ou tolerância diminuída aos carboidratos apresentam níveis tensionais mais elevados do que os acromegálicos sem estas comorbidades (57). A pressão arterial também foi mais elevada em pacientes diabéticos do que em euglicêmicos, tanto entre acromegálicos (curados ou não) quanto entre controles (3). Além disso, as alterações de tolerância à glicose influenciaram independentemente os níveis de pressão arterial de 24 horas, e a ausência de descenso noturno esteve associada à resistência insulínica em pacientes acromegálicos normotensos e hipertensos (3).

Os efeitos do tratamento da acromegalia sobre a hipertensão arterial ainda não estão bem esclarecidos, e os resultados descritos na literatura são conflitantes. Houve redução discreta da pressão arterial sistólica, mas não da diastólica, apenas nos pacientes curados da acromegalia, em um grupo de 33 acromegálicos e 33 controles (58). Em outro trabalho, houve redução tanto da pressão sistólica quanto da diastólica em acromegálicos com doença controlada e melhora do perfil circadiano da pressão em alguns desses pacientes (23). Em pacientes jovens, houve redução da pressão sistólica após 12 meses de tratamento com análogos da somatostatina (25). Com maior tempo de seguimento (cinco anos), verificou-se melhora dos níveis tensionais em repouso, mas não após exercício, e apenas em pacientes com doença controlada (22). Uma metanálise de 18 estudos avaliando o efeito de análogos da somatostatina sobre o coração não encontrou efeito significativo destes sobre o controle da pressão arterial (26). 


\section{ALTERAÇÕES METABÓLICAS E RISCO CARDIOVASCULAR}

A acromegalia sabidamente está associada a anormalidades do metabolismo lipídico. Há cerca de três décadas já se demonstrou que pacientes com acromegalia ativa tinham níveis de colesterol mais baixos e níveis de triglicerídeos mais altos do que os da população normal. Diversos estudos dedicaram-se a avaliar o metabolismo lipídico nos acromegálicos, com resultados, às vezes, conflitantes (3). Os níveis de colesterol total foram descritos como normais, aumentados ou até mesmo diminuídos na acromegalia; a lipoproteína a (Lp-a), as LDL pequenas e densas e os triglicerídeos estavam elevados na maioria dos estudos e o HDL foi descrito como inalterado ou diminuído (3). Outros marcadores metabólicos, como LCAT (lecitina-colesterol aciltransferase) e CETP (proteína transportadora de ésteres de colesterol), apresentaram-se diminuídos em pacientes acromegálicos (3). Em uma série de 42 pacientes com acromegalia, 57\% tinham perfil lipídico alterado, contra $51 \%$ dos controles, visto que a Lp-a era o único parâmetro lipídico alterado em $31 \%$ dos pacientes e em 7\% dos controles (59). Outra série com 20 pacientes ( 12 com acromegalia ativa e oito curados) encontrou níveis mais altos de Lp-a nos pacientes com doença em atividade, seguidos pelos pacientes curados; mesmo estes últimos ainda tinham níveis de Lp-a mais elevados do que os controles (60). Esse estudo sugere que o risco cardiovascular pode persistir significativamente elevado apesar do tratamento adequado da acromegalia, uma vez que os níveis de lipoproteínas aterogênicas se mantêm alterados em relação às pessoas normais. Vilar e cols. (61) encontraram níveis significativamente mais elevados de colesterol total, LDL, VLDL, triglicerídeos e Lp-a em pacientes acromegálicos do que em controles; quando comparados os acromegálicos com doença ativa e os com doença controlada, os primeiros tinham níveis mais elevados de VLDL, triglicerídeos e Lp-a. Nesse estudo, 56\% dos acromegáli$\cos \mathrm{em}$ atividade tinham hipertrigliceridemia, 66\% tinham níveis elevados de Lp-a e 80\% tinham LDL acima de $100 \mathrm{mg} / \mathrm{dL}$ (61). Por sua vez, os pacientes com acromegalia controlada tiveram frequências menores de hipertrigliceridemia (33\%), de elevação da Lp-a (30\%), e 83\% deles tinham LDL acima de $100 \mathrm{mg} / \mathrm{dL}$. Entre os controles, todos estes índices foram mais baixos, embora com significância estatística somente quando comparados aos acromegálicos com doença ativa.
Em geral, as alterações dos lipídeos nos acromegálicos são mais evidentes nos pacientes com alterações concomitantes no metabolismo glicêmico, embora não se possa excluir componentes genéticos, étnico-geográficos e dietéticos (3). Existem indícios de que o GH regula a síntese de $\mathrm{Lp}(\mathrm{a})$, bem como induz, quando em excesso, a redução da atividade da lipase lipoprotéica (LPL) (3). Além disso, em pacientes acromegálicos também está descrito aumento da concentração de partículas de LDL pequenas e densas (62), as quais estão sabidamente relacionadas ao aumento de complicações cardiovasculares em pacientes diabéticos e não-diabéticos. Diversas anormalidades na composição das partículas de HDL em acromegálicos são sugestivas de defeitos na ação da LCAT (lecitina-colesterol acil transferase) e de diminuição da proteína transportadora de fosfolipídios. Os defeitos na esterificação do colesterol e na atividade da proteína transportadora de fosfolipídios podem impedir o transporte reverso de colesterol, contribuindo para o aumento do risco cardiovascular (3).

O GH contrabalança os efeitos da insulina sobre a glicose, apesar de compartilhar com a insulina alguns efeitos anabólicos sobre o metabolismo protéico. Além disso, o GH modula as respostas teciduais à insulina, porquanto o excesso de GH pode causar resistência insulínica. A tolerância diminuída aos carboidratos e o diabetes melito estão freqüentemente associados à acromegalia. Os pacientes acromegálicos são insulinoresistentes, tanto no fígado quanto na periferia, apresentando hiperinsulinemia e aumento do turnover de glicose nos estados pós-absortivos (3). A prevalência de diabetes melito na acromegalia não está bem estabelecida, mas pode variar entre $19 \%$ e $56 \%$, conforme a série (63). Os estados intermediários do metabolismo glicídico (tolerância diminuída aos carboidratos e glicemia de jejum alterada) foram menos investigados até o momento, estimando prevalência do primeiro entre $16 \% \mathrm{e}$ $46 \%$ (63). Os principais fatores de risco para o desenvolvimento de diabetes melito na acromegalia foram os níveis de GH, a idade dos pacientes e o tempo de duração da doença (3) - fatores estes bastante semelhantes aos principais determinantes do aumento da mortalidade na acromegalia. Em estudo que avaliou a freqüência e os fatores de risco para intolerância à glicose na acromegalia, detectaram-se como seus principais determinantes a história familiar de diabetes e a coexistência de hipertensão arterial, além da associação entre diabetes e sexo feminino na população acromegálica estudada (63). 
Colao, citando Sonksen e cols., apresenta dois estágios no desenvolvimento do diabetes na acromegalia. O primeiro é hiperinsulinêmico, caracterizado por tolerância à glicose normal ou diminuída, com pico de insulina maior e mais rápido após sobrecarga de glicose, mas com descenso mais lento do que nos controles. $\mathrm{O}$ segundo caracteriza-se por resposta insulínica à glicose atrasada, com tolerância à glicose normal ou ligeiramente diminuída, alteração provavelmente reversível com o tratamento da acromegalia. Haveria, ainda, o terceiro estágio, irreversível mesmo com o tratamento da doença, caracterizado por resposta pancreática máxima no jejum sem o aumento esperado nas concentrações de insulina após sobrecarga de glicose (3). Ou seja, o excesso de GH induz insulino-resistência, que se manifesta inicialmente por aumento da insulina basal e da sua resposta à sobrecarga de glicose. Permanecendo não-tratado o hipersomatotropismo, essas alterações evoluem para hiperglicemia de jejum e diminuição dos níveis de insulina, tanto no jejum quanto, e principalmente, em resposta a aumentos da glicemia.

Os distúrbios do metabolismo glicêmico na acromegalia podem também estar associados a alterações no número de receptores de insulina disponíveis, na sua afinidade pela glicose ou em defeitos pós-receptor. Há trabalhos que demonstram ligação anômala da insulina ao seu receptor - achado este correlacionado com o nível de excesso de GH; também está descrita diminuição da concentração de receptores de insulina por célula (3).

Embora não tão extensamente estudadas quanto as alterações dos metabolismos lipídico e glicêmico, também podem ocorrer anormalidades do metabolismo protéico na acromegalia. O GH aumenta a força muscular e a retenção de nitrogênio, direta e indiretamente por meio da produção local e hepática de IGF-l (3). No entanto, a maior parte das modificações no metabolismo das proteínas ocorre por meio dos efeitos do GH e do IGF-1 sobre a função renal. A hiperfiltração glomerular é característica da acromegalia. A excreção urinária de albumina é mais elevada em pacientes acromegálicos do que em controles, sugerindo influência do eixo somatotrófico sobre a albuminúria (3). Baldelli e cols. (64) avaliaram a albuminúria de 24 horas em 74 acromegálicos e 50 controles, encontrando microalbuminúria em $55 \%$ dos pacientes e em nenhum dos controles; os pacientes hipertensos e os com alterações do metabolismo glicêmico tinham níveis de microalbuminúria mais elevados que os pacientes normotensos ou normoglicêmicos. Dado que a albuminúria pode ser considerada marcadora de disfunção endotelial, esse achado corrobora o aumento do risco cardiovascular a que os acromegálicos estão expostos.

As alterações do metabolismo glicêmico são passíveis de melhora ou reversão com o adequado tratamento da doença. Já na década de 1960 verificou-se que a remoção do tumor produtor de GH ocasionava melhora da tolerância à glicose e mesmo "cura" do diabetes. Posteriormente, o advento dos análogos da somatostatina suscitou dúvidas quanto à sua capacidade de restabelecer a normalidade do metabolismo glicêmico nos acromegálicos, uma vez que esses fármacos inibem a secreção pancreática de insulina. No entanto, essa inibição mostrou-se, na prática, menor do que se supunha inicialmente; além disso, a melhora da resistência insulínica induzida pelos análogos da somatostatina contrabalança o efeito inibitório sobre o pâncreas. O pegvisomanto, antagonista do GH, mostrou ser capaz de melhorar a sensibilidade à insulina e o metabolismo dos carboidratos na acromegalia. Rose e Clemmons (65) demonstraram redução dos níveis basais de insulina e glicose após tratamento com pegvisomanto, sem modificação do peso corporal dos pacientes. Van der Lely e cols. (66) encontraram resultados semelhantes, com número bem maior de pacientes.

A maior parte dos estudos que avaliaram as mudanças de perfil lipídico após o tratamento da acromegalia encontrou redução de lipídeos aterogênicos, embora haja resultados negativos. Em geral, o octreotide melhora o perfil lipídico sem, no entanto, modificar as LDL pequenas e densas, que são particularmente aterogênicas. No estudo de van der Lely (66), não houve modificação significativa de colesterol total ou triglicerídeos após o tratamento com pegvisomanto. No trabalho de Sesmilo e cols. (67), após 18 meses de pegvisomanto, houve aumento do colesterol total e dos triglicerídeos, enquanto a Lp-a diminuiu. Nesse mesmo estudo, houve aumento dos níveis de proteína C-reativa ultrasensível após o tratamento, achado este que também ocorreu no estudo de Vilar e cols. (61). Esse achado poderia representar tanto o efeito direto dos níveis de GH sobre esta proteína quanto o reflexo de possível deficiência relativa de GH nos pacientes tratados. Em estudo com 20 acromegálicos, avaliados antes e após o pegvisomanto, encontraram-se níveis iniciais reduzidos de colesterol total e LDL, os quais aumentaram após o tratamento (68). Já a Lp-a, inicialmente elevada na maioria dos pacientes, diminuiu com o tratamento. 
Sumarizando, a acromegalia se faz acompanhar de diversas alterações metabólicas, especialmente dos metabolismos glicêmico e lipídico, as quais, isoladamente ou associadas, conferem perfil de risco cardiovascular elevado aos pacientes. Essas alterações são passíveis de melhora, ao menos em parte, com o controle bioquímico da doença, embora ainda não haja dados suficientes para saber se isso se reflete em melhora da expectativa de vida dos pacientes a longo prazo.

\section{CONCLUSÕES}

A acromegalia provoca considerável impacto sobre a mortalidade e a qualidade de vida dos pacientes afetados. Em diversas coortes de acromegálicos, as taxas de mortalidade são mais elevadas do que as da população geral (9-11,45,69). As razões para esse aumento da mortalidade se devem, especialmente, às complicações decorrentes do excesso crônico de GH e IGF-1. Entre elas, uma das que mais causam aumento do número de mortes é a doença cardiovascular. $\mathrm{O}$ espectro da doença cardiovascular no acromegálico compreende hipertrofia ventricular esquerda, disfunções diastólica e sistólica, fibrose miocárdica, valvulopatias, arritmias, hipertensão arterial, dislipidemia e alteração no metabolismo dos carboidratos. Essas entidades respondem conjuntamente por até $50 \%$ das mortes de pacientes acromegálicos, dependendo do estudo $(9,10,45)$. Holdaway, Rajasoorya e Gamble (10) apontam como principais determinantes de mortalidade na acromegalia os níveis de GH acima de $2 \mu \mathrm{g} / \mathrm{L}$ no último seguimento, a idade do paciente, a presença de hipertensão arterial e a duração estimada da doença antes do tratamento; o GH foi preditor independente de mortalidade.

Com o desenvolvimento de formas de tratamento mais efetivas, como a cirurgia transesfenoidal, as medicações que atuam sobre o GH e o IGF-l e as novas modalidades de radioterapia, vêm sendo obtidos melhores índices de remissão da acromegalia. Estes índices poderão se refletir na diminuição do risco de mortalidade dos pacientes a longo prazo, embora mesmo entre pacientes que obtenham cura bioquímica da doença não ocorra a normalização correspondente das taxas de mortalidade (11). Uma possível explicação é o fato de que as alterações provocadas pela longa exposição aos níveis elevados de GH/IGF-1 nem sempre são revertidas com a cura da acromegalia. Isso é particularmente verdadeiro com a doença cardiovascular do paciente acromegálico, em especial com a hipertensão arterial.
Ou seja, pacientes curados, sob o ponto de vista hormonal, podem permanecer ainda com alterações cardiovasculares importantes e sabidamente agregadoras de risco de mortalidade.

A despeito de boa parte da fisiopatologia destas alterações não estar totalmente elucidada, fica evidente que a diminuição da morbimortalidade cardíaca da acromegalia inclui o adequado controle dos níveis de GH e IGF-1. Provavelmente o maior benefício que se pode oferecer aos pacientes acromegálicos nesse sentido é diagnosticar, cada vez mais precocemente, a doença, evitando-se, assim, a progressão da doença cardiovascular. Já se avançou muito nesta direção, em particular após o refinamento dos critérios de cura, mas a acromegalia continua sendo doença de diagnóstico relativamente tardio.

É importante ressaltar aqui o papel do "supertratamento" da acromegalia no desenvolvimento do perfil cardiovascular anômalo de que se tratou até agora. A conjugação de cirurgia, tratamento medicamentoso (análogos da somatostatina, principalmente) e radioterapia pode provocar a diminuição da secreção de $\mathrm{GH}$, além dos limites considerados normais, não raro transformando acromegálicos em pacientes deficientes de GH. Assim, parte do complexo "alterações endoteliais - cardiopatia isquêmica - dislipidemia - hipertensão" pode ter origem não apenas nos efeitos tardios do excesso prolongado de $\mathrm{GH}$, mas, também na deficiência deste hormônio provocada pelo tratamento da acromegalia. É bem reconhecido o espectro de doença cardiovascular decorrente da deficiência de $\mathrm{GH}$, que se caracteriza fundamentalmente por alterações do perfil liṕ́dico (hipertrigliceridemia, elevado LDL), aterosclerose prematura, resistência insulínica e performance cardíaca reduzida (14).

Por outro lado, a diminuição da morbimortalidade cardiovascular do paciente acromegálico não se resume ao controle bioquímico da doença. Os pacientes tratados nos quais não ocorre a esperada redução dos níveis pressóricos, da hipertrofia cardíaca ou dos lipídeos séricos, necessitam de intervenções agressivas nestes fatores de risco e acompanhamento constante. Isso é válido mesmo após obtida a cura da acromegalia, já que fica claro que esta, isoladamente, não é capaz de trazer o paciente de volta aos patamares de risco cardíaco anteriores à doença. Assim, enquanto não é efetivamente possível efetuar "intervenção precoce", torna-se fundamental intervir agressivamente nos fatores cardiovasculares aqui abordados. De maneira análoga ao que se faz 
atualmente no acompanhamento do diabetes melito, tratar agressivamente a hipertensão arterial, seguir em longo prazo a função cardíaca, monitorar e tratar as dislipidemias e as alterações da glicose são estratégias relativamente simples e que podem eventualmente contribuir para reduzir o excesso de mortalidade que os acromegálicos tratados continuam a sofrer.

Os autores declaram não haver conflitos de interesse científico neste artigo.

\section{REFERÊNCIAS}

1. Holdaway IM, Rajasoorya C. Epidemiology of acromegaly. Pituitary. 1999;2(1):29-41.

2. Melmed S. Acromegaly. In: Melmed S, ed. The Pituitary. Massachusets: Blackwell Publishing. 2002. p.419-54.

3. Colao A, Ferone D, Marzullo P, Lombardi G. Systemic complications of acromegaly: epidemiology, pathogenesis, and management. Endocr Rev. 2004;25(1):102-52.

4. Melmed S. Medical progress: acromegaly. N Engl J Med. 2006;355(24):2558-73.

5. Vitale G, Pivonello R, Lombardi G, Colao A. Cardiac abnormalities in acromegaly. Pathophysiology and implications for management. Treat Endocrinol. 2004;3(5):309-18.

6. Clayton RN. Cardiovascular function in acromegaly. Endocr Rev. 2003;24(3):272-7.

7. Otsuki M, Kasayama S, Yamamoto H, Saito H, Sumitani S, Kouhara $\mathrm{H}$, et al. Characterization of premature atherosclerosis of carotid arteries in acromegalic patients. Clin Endocrinol (Oxf). 2001;54(6):791-6.

8. Katznelson L. Current thinking on the management of the acromegalic patient. Curr Opin Endocrinol Diabetes Obes. 2007;14(4):311-6.

9. Mestron A, Webb SM, Astorga R, Benito P, Catala M, Gaztambide $S$, et al. Epidemiology, clinical characteristics, outcome, morbidity and mortality in acromegaly based on the Spanish Acromegaly Registry (Registro Espanol de Acromegalia, REA). Eur J Endocrinol. 2004;151(4):439-46.

10. Holdaway IM, Rajasoorya RC, Gamble GD. Factors influencing mortality in acromegaly. J Clin Endocrinol Metabolism. 2004;89(2):667-74.

11. Dekkers OM, Biermasz NR, Pereira AM, Romijn JA, Vandenbroucke JP. Mortality in acromegaly: a metaanalysis. J Clin Endocrinol Metabolism. 2008;93(1):61-7.

12. Casagrande A, Czepielewski MA. Growth hormone (GH) and IGF-I assays: methodological aspects and its implications in acromegaly diagnosis and follow-up. Arq Bras Endocrinol Metabol. 2007;51(4):511-9.

13. Minniti G, Jaffrain-Rea ML, Moroni C, Baldelli R, Ferretti E, Cassone R, et al. Echocardiographic evidence for a direct effect of GH/IGF-I hypersecretion on cardiac mass and function in young acromegalics. Clin Endocrinol (Oxf). 1998;49(1):101-6.

14. Colao A, Marzullo P, Di Somma C, Lombardi G. Growth hormone and the heart. Clinical Endocrinology. 2001;54(2):137-54.

15. Sacca L, Cittadini A, Fazio S. Growth hormone and the heart. Endocr Rev. 1994;15(5):555-73.

16. Vitale G, Galderisi M, Pivonello R, Spinelli L, Ciccarelli A, de Divitiis $\mathrm{O}$, et al. Prevalence and determinants of left ventricular hyper- trophy in acromegaly: impact of different methods of indexing left ventricular mass. Clin Endocrinol (Oxf). 2004;60(3): 343-9.

17. Fazio S, Cittadini A, Biondi B, Palmieri EA, Riccio G, Bone F, et al. Cardiovascular effects of short-term growth hormone hypersecretion. J Clin Endocrinol Metabolism. 2000;85(1):179-82.

18. Casini AF, Araujo PB, Fontes R, Xavier SS, Gadelha MR. Cardiac morphology and performance alterations and analysis of determinant factors of left ventricular hypertrophy in 40 patients with acromegaly. Arq Bras Endocrinol Metabol. 2006; 50(1):82-90.

19. Lopez-Velasco R, Escobar-Morreale HF, Vega B, Villa E, Sancho $\mathrm{JM}$, Moya-Mur JL, et al. Cardiac involvement in acromegaly: specific myocardiopathy or consequence of systemic hypertension? J Clin Endocrinol Metabolism. 1997;82(4):1047-53.

20. Swearingen B, Barker FG, 2nd, Katznelson L, Biller BM, Grinspoon S, Klibanski A, et al. Long-term mortality after transsphenoidal surgery and adjunctive therapy for acromegaly. J Clin Endocrinol Metabolism. 1998;83(10):3419-26.

21. Baldwin A, Cundy T, Butler J, Timmis AD. Progression of cardiovascular disease in acromegalic patients treated by external pituitary irradiation. Acta Endocrinol (Copenh). 1985;108(1):26-30.

22. Colao A, Cuocolo A, Marzullo P, Nicolai E, Ferone D, Della Morte $A M$, et al. Is the acromegalic cardiomyopathy reversible? Effect of 5-year normalization of growth hormone and insulinlike growth factor I levels on cardiac performance. J Clin Endocrinol Metabolism. 2001;86(4):1551-7.

23. Minniti G, Moroni C, Jaffrain-Rea ML, Esposito V, Santoro A, Affricano $C$, et al. Marked improvement in cardiovascular function after successful transsphenoidal surgery in acromegalic patients. Clin Endocrinol (Oxf). 2001;55(3):307-13.

24. Ciulla MM, Epaminonda $P$, Paliotti R, Barelli MV, Ronchi $C$, Cappiello V, et al. Evaluation of cardiac structure by echoreflectivity analysis in acromegaly: effects of treatment. Eur $\mathrm{J}$ Endocrinol. 2004;151(2):179-86.

25. Colao A, Marzullo P, Cuocolo A, Spinelli L, Pivonello R, Bonaduce $D$, et al. Reversal of acromegalic cardiomyopathy in young but not in middle-aged patients after 12 months of treatment with the depot long-acting somatostatin analogue octreotide. Clin Endocrinol (Oxf). 2003;58(2):169-76.

26. Maison P, Tropeano Al, Macquin-Mavier I, Giustina A, Chanson P. Impact of somatostatin analogs on the heart in acromegaly: a metaanalysis. J Clin Endocrinol Metabolism. 2007;92(5):1743-7.

27. Pivonello R, Galderisi M, Auriemma RS, De Martino MC, Galdiero M, Ciccarelli A, et al. Treatment with growth hormone receptor antagonist in acromegaly: effect on cardiac structure and performance. J Clin Endocrinol Metabolism. 2007; 92(2): 476-82.

28. Kahaly G, Olshausen KV, Mohr-Kahaly S, Erbel R, Boor S, Beyer J, et al. Arrhythmia profile in acromegaly. Eur Heart $\mathrm{J}$. 1992;13(1):51-6.

29. Fatti LM, Scacchi M, Lavezzi E, Pecori Giraldi F, De Martin M, Toja $\mathrm{P}$, et al. Effects of treatment with somatostatin analogues on QT interval duration in acromegalic patients. Clin Endocrinol (Oxf). 2006;65(5):626-30.

30. Herrmann BL, Bruch C, Saller B, Ferdin S, Dagres N, Ose C, et al. Occurrence of ventricular late potentials in patients with active acromegaly. Clin Endocrinol (Oxf). 2001;55(2):201-7.

31. Lombardi G, Colao A, Marzullo P, Biondi B, Palmieri E, Fazio S. Improvement of left ventricular hypertrophy and arrhythmias after lanreotide-induced GH and IGF-I decrease in acromegaly. A prospective multi-center study. J Endocrinol Invest. 2002;25 (11):971-6. 
32. Colao A, Spinelli L, Marzullo P, Pivonello R, Petretta M, Di Somma $\mathrm{C}$, et al. High prevalence of cardiac valve disease in acromegaly: an observational, analytical, case-control study. J Clin Endocrinol Metabolism. 2003;88(7):3196-201.

33. Lie JT. Pathology of the heart in acromegaly: anatomic findings in 27 autopsied patients. Am Heart J. 1980;100(1):41-52.

34. Ohtsuka G, Aomi S, Koyanagi H, Tsukui H, Tomizawa Y, Hashimoto $A$, et al. Heart valve operation in acromegaly. Ann Thorac Surg. 1997;64(2):390-3.

35. Pereira AM, van Thiel SW, Lindner JR, Roelfsema F, van der Wall EE, Morreau H, et al. Increased prevalence of regurgitant valvular heart disease in acromegaly. J Clin Endocrinol Metabolism. 2004;89(1):71-5.

36. van der Klaauw AA, Bax JJ, Roelfsema F, Bleeker GB, Holman ER, Corssmit EP, et al. Uncontrolled acromegaly is associated with progressive mitral valvular regurgitation. Growth Horm IGF Res. 2006 Apr;16(2):101-7.

37. Zanettini R, Antonini A, Gatto G, Gentile R, Tesei S, Pezzoli G. Valvular heart disease and the use of dopamine agonists for Parkinson's disease. N Engl J Med. 2007;356(1):39-46.

38. Schade R, Andersohn F, Suissa S, Haverkamp W, Garbe E. Dopamine agonists and the risk of cardiac-valve regurgitation. $\mathrm{N}$ Engl J Med. 2007;356(1):29-38.

39. Ross R. Atherosclerosis-an inflammatory disease. New England Journal of Medicine. 1999;340(2):115-26.

40. Chanson P, Megnien JL, del Pino M, Coirault C, Merli I, Houdouin $L$, et al. Decreased regional blood flow in patients with acromegaly. Clin Endocrinol (Oxf). 1998;49(6):725-31.

41. Maison P, Demolis P, Young J, Schaison G, Giudicelli JF, Chanson $\mathrm{P}$. Vascular reactivity in acromegalic patients: preliminary evidence for regional endothelial dysfunction and increased sympathetic vasoconstriction. Clin Endocrinol (Oxf). 2000;53( 4):445-51.

42. Brevetti G, Marzullo P, Silvestro A, Pivonello R, Oliva G, di Somma $\mathrm{C}$, et al. Early vascular alterations in acromegaly. J Clin Endocrinol Metabolism. 2002;87(7):3174-9.

43. Schiavon F, Maffei P, Martini C, De Carlo E, Fais C, Todesco S, et al. Morphologic study of microcirculation in acromegaly by capillaroscopy. J Clin Endocrinol Metabolism. 1999;84(9):3151-5.

44. Colao A, Spiezia S, Cerbone G, Pivonello R, Marzullo P, Ferone $D$, et al. Increased arterial intima-media thickness by B-M mode echodoppler ultrasonography in acromegaly. Clin Endocrinol (Oxf). 2001;54(4):515-24.

45. Kauppinen-Makelin R, Sane T, Reunanen A, Valimaki MJ, Niskanen $\mathrm{L}$, Markkanen $\mathrm{H}$, et al. A nationwide survey of mortality in acromegaly. J Clin Endocrinol Metabolism. 2005;90(7):4081-6.

46. Cannavo S, Almoto B, Cavalli G, Squadrito S, Romanello G, Vigo MT, et al. Acromegaly and coronary disease: an integrated evaluation of conventional coronary risk factors and coronary calcifications detected by computed tomography. J Clin Endocrinol Metabolism. 2006;91(10):3766-72.

47. Bogazzi F, Battolla L, Spinelli C, Rossi G, Gavioli S, Di Bello V, et al. Risk factors for development of coronary heart disease in patients with acromegaly: a five-year prospective study. J Clin Endocrinol Metabolism. 2007;92(11):4271-7.

48. Vitale G, Pivonello R, Auriemma RS, Guerra E, Milone F, Savastano $S$, et al. Hypertension in acromegaly and in the normal population: prevalence and determinants. Clin Endocrinol (Oxf). 2005;63(4):470-6.

49. Mulatero P, Veglio F, Maffei P, Bondanelli M, Bovio S, Daffara F, et al. CYP11B2-344T/C gene polymorphism and blood pressu- re in patients with acromegaly. J Clin Endocrinol Metabolism. 2006;91(12):5008-12

50. Andreassen M, Faber J, Vestergaard H, Kistorp C, Kristensen LO. N-terminal pro-B-type natriuretic peptide in patients with growth hormone disturbances. Clin Endocrinol (Oxf). 2007; 66(5):619-25.

51. Smith JC, Lane H, Davies N, Evans LM, Cockcroft J, Scanlon $\mathrm{MF}$, et al. The effects of depot long-acting somatostatin analog on central aortic pressure and arterial stiffness in acromegaly. $\mathrm{J}$ Clin Endocrinol Metabolism. 2003;88(6):2556-61.

52. Rizzoni D, Porteri E, Giustina A, De Ciuceis C, Sleiman I, Boari $\mathrm{GE}$, et al. Acromegalic patients show the presence of hypertrophic remodeling of subcutaneous small resistance arteries. Hypertension. 2004;43(3):561-5.

53. Rio GD, Velardo A, Mascadri C, Zalteri G, Papi G, Menozzi R, et al. Baseline and stimulated catecholamine secretion in normotensive patients with active acromegaly: acute effects of continuous octreotide infusion. Eur J Endocrinol. 2000;142(2):179-86.

54. Bondanelli M, Ambrosio MR, Franceschetti P, Margutti A, Trasforini G, Degli Uberti EC. Diurnal rhythm of plasma catecholamines in acromegaly. J Clin Endocrinol Metabolism. 1999;84(7):2458-67.

55. Jaffrain-Rea ML, Moroni C, Baldelli R, Battista C, Maffei P, Terzolo $\mathrm{M}$, et al. Relationship between blood pressure and glucose tolerance in acromegaly. Clin Endocrinol (Oxf). 2001;54(2):189-95.

56. Feld S, Hirschberg R. Growth hormone, the insulin-like growth factor system, and the kidney. Endocr Rev. 1996;17(5):423-80.

57. Colao A, Baldelli R, Marzullo P, Ferretti E, Ferone D, Gargiulo $\mathrm{P}$, et al. Systemic hypertension and impaired glucose tolerance are independently correlated to the severity of the acromegalic cardiomyopathy. J Clin Endocrinol Metabolism. 2000;85(1):193-9.

58. Ronchi CL, Varca V, Beck-Peccoz P, Orsi E, Donadio F, Baccarelli $A$, et al. Comparison between six-year therapy with long-acting somatostatin analogs and successful surgery in acromegaly: effects on cardiovascular risk factors. J Clin Endocrinol Metabolism. 2006;91(1):121-8.

59. Maffei P, Sicolo N, Plebani M. Lipoprotein(a) in acromegaly. Ann Intern Med. 1999;130(6):537-8.

60. Maldonado Castro GF, Escobar-Morreale HF, Ortega H, GomezCoronado D, Balsa Barro JA, Varela C, et al. Effects of normalization of $\mathrm{GH}$ hypersecretion on lipoprotein(a) and other lipoprotein serum levels in acromegaly. Clin Endocrinol (Oxf). 2000;53(3):313-9.

61. Vilar L, Naves LA, Costa SS, Abdalla LF, Coelho CE, Casulari LA. Increase of classic and nonclassic cardiovascular risk factors in patients with acromegaly. Endocr Pract. 2007;13 (4):363-72.

62. Arosio M, Macchelli S, Rossi CM, Casati G, Biella O, Faglia G. Effects of treatment with octreotide in acromegalic patients-a multicenter Italian study. Italian Multicenter Octreotide Study Group. Eur J Endocrinol. 1995;133(4):430-9.

63. Kreze A, Kreze-Spirova E, Mikulecky M. Risk factors for glucose intolerance in active acromegaly. Braz J Med Biol Res. 2001;34(11):1429-33.

64. Baldelli R, De Marinis L, Bianchi A, Pivonello R, Gasco V, Auriemma $R$, et al. Microalbuminuria and insulin sensitivity in patients with Gh-secreting pituitary tumor. J Clin Endocrinol Metabolism. 2008;93(3):710-4.

65. Rose DR, Clemmons DR. Growth hormone receptor antagonist improves insulin resistance in acromegaly. Growth Horm IGF Res. 2002;12(6):418-24. 
66. van der Lely AJ, Hutson RK, Trainer PJ, Besser GM, Barkan AL, Katznelson $L$, et al. Long-term treatment of acromegaly with pegvisomant, a growth hormone receptor antagonist. Lancet. 2001;358(9295):1754-9.

67. Sesmilo G, Fairfield WP, Katznelson L, Pulaski K, Freda PU, Bonert V, et al. Cardiovascular risk factors in acromegaly before and after normalization of serum IGF-I levels with the GH antagonist pegvisomant. J Clin Endocrinol Metabolism. 2002;87 (4):1692-9.

68. Parkinson C, Drake WM, Wieringa G, Yates AP, Besser GM, Trainer PJ. Serum lipoprotein changes following IGF-I normalization using a growth hormone receptor antagonist in acromegaly. Clin Endocrinol (Oxf). 2002;56(3):303-11.
69. Orme SM, McNally RJ, Cartwright RA, Belchetz PE. Mortality and cancer incidence in acromegaly: a retrospective cohort study. United Kingdom Acromegaly Study Group. J Clin Endocrinol Metabolism. 1998;83(8):2730-4.

Endereço para correspondência:

Daniela Fedrizzi

Serv. Endocrinologia do Hospital de Clínicas de Porto Alegre Rua Ramiro Barcellos, 2350, prédio 12, $4^{\circ}$ andar

90035-003 Porto Alegre RS

E-mail: danifedrizzi@hotmail.com 